\title{
Benign Migratory Glossitis: Case Report and Literature Review
}

\author{
Sarfaraz Khan ${ }^{1 *}$, Syed AsifHaider Shah ${ }^{2}$, Tanveer Ahmed Mujahid ${ }^{3}$ and Muhammad Ishaq ${ }^{4}$ \\ ${ }^{1}$ Consultant Oral and Maxillofacial Surgeon, Pak Field Hospital Darfur, Sudan \\ ${ }^{2}$ MDC Gujranwala, Pakistan \\ ${ }^{3}$ Consultant Dermatologist, Pak Field Hospital Darfur, Sudan
}

${ }^{4}$ Registrar Dermatologist, Pak Field Hospital Darfur, Sudan

Received: October 25, 2017; Published: October 31, 2017

*Corresponding author: Sarfaraz Khan, Consultant Oral and Maxillofacial Surgeon, Pakistan Field Hospital Darfur, Sudan, Tel: +92 300 9323960; Email: smilemechanic@hotmail.com

\section{Abstract}

Benign migratory Glossitis (BMG) is a benign, usually asymptomatic mucosal lesion of dorsal surface of the tongue, characterized by depapillated erythematous patches separated by white irregular borders. Etiology of BMG is unknown. Risk factors include psoriasis, fissured tongue, diabetes mellitus, hypersensitivity and psychological factors. We report BMG in an Egyptian soldier of UN peace keeping force, with stress as a possible etiological factor and provide literature review of this disorder.

Keywords: Geographic tongue; Benign migratory Glossitis; Erythema migrans

\section{Introduction}

Benign migratory Glossitis (BMG) is a benign, immunemediated, chronic inflammatory lesion of unknown etiology, usually characterized by asymptomatic erythematous patches with whitish margins across the surface of the tongue. This condition is also known as geographic tongue, erythema migrans, Glossitis exfoliativa and wandering rash of the tongue. The central erythematous patch represents atrophy of the filiform papillae. The white border is composed of regenerating filiform papillae and a mixture of keratin and neutrophil aggregates within the epithelium. The most frequently reported prevalence is $1-2.5 \%$ with no gender predilection. It commonly occurs on the tip, lateral borders, and dorsum of the tongue; extending sometimes to the ventral surface as well. BMG shows periods of exacerbation and remission with recovery in one area and appearance in other area; thus explaining the typical migratory nature of this lesion [1-3].

Etiology of BMG is not well established. Various studies have found association of BMG with psoriasis, diabetes mellitus, Reiter's syndrome, Down's syndrome, pregnancy, psychological factors, genetic factors, hypersensitivity, fissured tongue [3] and consumption of oral contraceptive pills and lithium carbonate [4]. The diagnosis is based on history and clinical presentation. Though usually asymptomatic in nature; pain and burning sensation in the affected area of the tongue has been reported on consumption of spicy/salty food and/or alcoholic drinks [4,5].The lesion typically changes its shape with time owing to the change in pattern of depapillation.

Similar lesions may also be seen in atrophic candidiasis, local chemical or mechanical trauma, drug induced reactions, psoriasis and atrophic lichen planus [6]. Asymptomatic BMG needs no therapy. Treatment of symptomatic BMG aims at provision of symptomatic relief by means of topical application of corticosteroids, local anesthetic agents, anti-inflammatory mouthwashes and systemic use of antihistamines and Zinc supplements [7]. We report a 26-year-old patient, an Egyptian soldier of UN peace keeping force, suffering from BMG; with stress as a possible etiological factor.

\section{Case Report}

A 26-year-old male patient, an Egyptian soldier performing his duties in United Nations-African Union Mission in Darfur (UNAMID), reported to Pakistani Field Hospital Darfur, Sudan; complaining of pain and burning sensation in his tongue for last two months. The patient was asymptomatic two months back when he developed burning sensation in his tongue on taking spicy food and hot drinks. After a few days the severity of burning increased in response to the same stimuli. In addition to it, he developed mild continuous pain and discomfort in tongue which aggravated during speech and mastication. He took multivitamin tablets and systemic antifungal 
medication (capsules) on the advice of a general practitioner but was not relieved of his symptoms. He also complained of disturbed sleep and loss of appetite. The patient related his symptoms with composite filling of his upper molar tooth which had been done one month before appearance of his symptoms.

His medical history was not significant. Currently he was not taking any medication and denied allergy to any medication or food item. According to the patient, no member of his family ever experienced such problem. He was nonalcoholic and remained nonsmoker until recently when he started smoking since last two weeks, about 10 cigarettes per day, without any known reason. He was unmarried and engaged to a lady about 18 months back. His marriage was delayed because of his job/service commitments. He was the eldest son of the family, having two sisters, two brothers and two parents; all dependent upon him. In mission area, his job was procurement and logistics management.

On clinical examination, he was a young male of average built and height with mesofacial profile. TMJ examination and mouth opening were normal. Intraoral examination showed satisfactory oral hygiene with all teeth intact. There were wear facets on lower anterior teeth. Examination of tongue revealed characteristic morphological features of geographic tongue. Multiple flat, smooth, erythematous patches of depapillated mucosa with yellowish white, slightly elevated, irregular peripheral borders were visible on dorsum and lateral margins of the tongue (Figure 1). No ulceration, bleeding or pus discharge was observed. Systemic examinations were unremarkable.

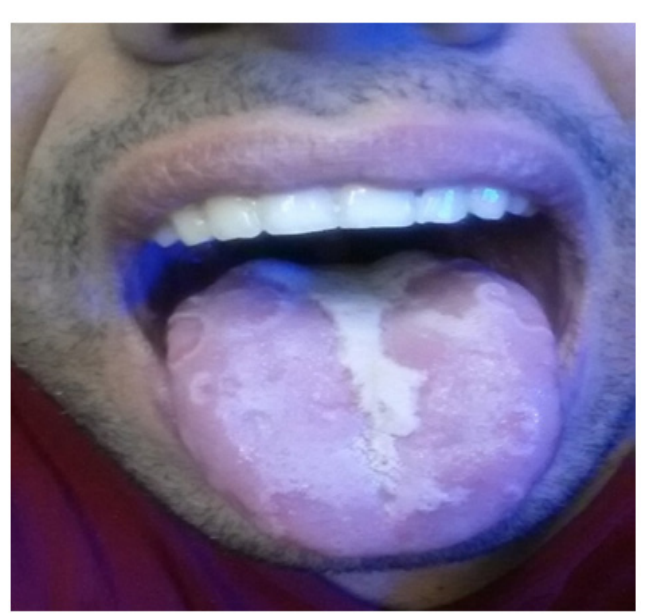

Figure 1: Initial clinical presentation of geographic tongue.

As the clinical diagnosis was straightforward, a biopsy was not deemed necessary. Other investigations included blood complete picture with RBC indices, serum glucose level, serum folate, vitamin B12 and albumin level and total Iron binding capacity; to distinguish from Glossitis associated with anemia or other nutritional deficiencies. Patient was reassured of the benign nature of the lesion. For symptomatic relief, he was advised topical application of Triamcinolone acetonide (Kenalog in orabaseointment) and use of Benzydamine mouth wash for 10 days. He was also advised tablet Paroxetine, $10 \mathrm{mg}$ once daily for 3 months, by our Psychiatrist. The patient was reviewed after 2 weeks who reported significant reduction in severity of his symptoms with only mild discomfort during eating. On examination of the tongue, the peripheral white zones on lateral margins of the tongue had disappeared. (Figure 2) Patient was advised to observe fortnightly follow up visits for the rest of his stay in mission area.

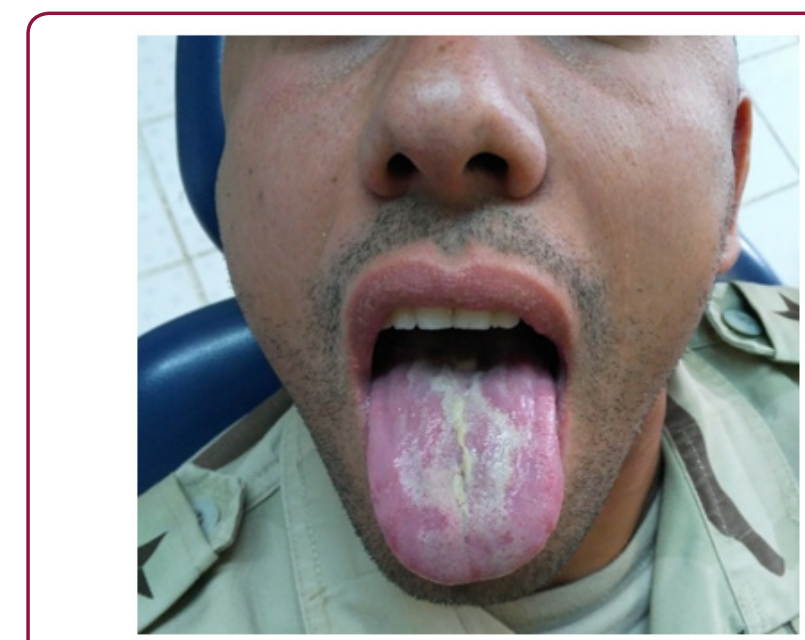

Figure 2: 10 days post-treatment.

\section{Discussion}

BMG is one of the most prevalent oral mucosal lesions (1$2.5 \%$, usually adults) [7]. Our patient demonstrated typical appearance and location of BMG, though it may occur on other sites of oral mucosa than on the dorsum of the tongue where it is called geographic stomatitis $[7,8]$. BMG is usually asymptomatic. However, itching, foreign body sensaרtion, mild pain and mild to severe burning sensation, which may occasionally interfere with eating or sleep $\neg$ ing, have also been reported. The severity of symptoms varies at different times, depending on the disease activity. Our patient reported disturbed sleep owing to pain and burning sensations which aggravated on taking spicy food and hot drinks. Cancer phobia has been reported in patients of BMG, due to which they, sometimes, seek medical help even in asymptomatic cases [2].

Etiology of BMG could not be established in our patient. No association was found between his disease and the reported risk factors like inheritance, diabetes mellitus, nutritional deficiency, Reiter's syndrome etc. His family and past medical history was not contributory. Allergy has been proposed as a major causative factor in BMG. An association has also been reported between the BMG and asthma, eczema, hay fever, elevated serum immunoglobulin $\mathrm{E}$ (IgE) and atopic patients [4,9]. Our patient denied allergy to any medication Various studies have demonstrated an association between BMG and psoriasis on the bases of microscopic similarity between the two conditions and the presence of a common genetic marker, HLA-C*06.Its prevalenceina population of psoriasis patients is approximately $5 \%[1,4,8]$. Our patient had no psoriasis.

An association between BMG and the fissured tongue has been reported in literature $[1,4,10,11]$. The fissured tongue is an asymptomatic, benign condition characterized by fissures or grooves on dorsal surface of the tongue. Fissured tongue should be interpreted as an end stage of BMG.7 In our patient; BMG was not 
accompanied by fissured tongue. Various studies support the role of emotional stress, as a causative agent, in the development and/or exacerbation of BMG $[4,12,13]$. Redman et al. [12] investigated the association of emotional stress and BMG in a population of students and found evidence to suggest emotional stress as an etiological factor in BMG. They also suggested that in a person with signs of psychological disturbance, the appearance of geographic tongue might be considered as additional evidence of the disturbance.

After exclusion of other risk factors, as mentioned in the literature, we suggest work/emotional stress to be a factor in development and/or exacerbation of BMG in our patient. Being the eldest son of the family with lot of familial responsibilities on his shoulders, who was away from his family for last 10 months, performing his duties in a Foreign country; coupled with requirements of commitment to his job might have put him under lot of stress which could develop BMG in our patient. Moreover; there is a possibility of the presence of an asymptomatic lesion which was diagnosed only after the appearance and exacerbation of symptoms during the period of stress.

\section{Conclusion}

In this case the lesion was readily identifiable clinically so biopsy was not considered. If biopsy is considered, it should involve the peripheral zone to include the typical microscopic features of this lesion; parakeratosis, acanthosis and sub epithelial Tlymphocytic inflammation [4]. Symptomatic treatment with topical corticosteroids, topical local anesthetic agents, antiinflammatory mouthwashes, antihistamines and anxiolytic dugs is documented in literature [6]. Our patient responded well to a 10 days course of topical corticosteroids (triamcinoloneacetonide) with significant reduction of his symptoms and disappearance of Peripheral zone of the lesion, which is a sign of recovering mucosa $[6,7]$. In conclusion, BMG is a completely benign mucosal lesion that shows periods of exacerbation and remission. In asymptomatic cases, its diagnosis is important to relieve cancer anxiety of the patient. In symptomatic cases, symptomatic treatment should be provided by use of corticosteroids along with correction of the cause. Possible role of stress in development of symptomatic BMG has been observed in this case report which may be confirmed by further investigations.

\section{References}

1. Picciani BL, Domingos TA, Teixeira-Souza T, Santos Vde C, Gonzaga HF, et al. (2016) Geographic tongue and psoriasis: clinical, histopathological, immunohisto chemical and genetic correlation - a literature review. An Bras Dermatol 91(4): 410-421.

2. Najafi S, Gholizadeh N, Akhavan Rezayat E, Kharrazifard MJ (2016) Treatment of Symptomatic Geographic Tongue with Triamcinolone Acetonide Alone and in Combination with Retinoic Acid: A Randomized Clinical Trial. J Dent (Tehran) 13(1): 23-28.

3. Goswami M, Verma A, Verma M (2012) benign migratory glossitis with fissured tongue. J Indian Soc Pedod Prev Dent 30(2): 173-175.

4. Honarmand M, Farhad ML, Shirzaiy M, Sehhatpour M (2013) Geographic Tongue and Associated Risk Factors among Iranian Dental Patients. Iran J Public Health 42(2): 215-219.

5. Jahanbani J, Sandvik L, Lyberg T, Ahlfors E (2009) Evaluation of oral mucosal lesions in 598 referred Iranian patients. Open Dent J 3:42-47.

6. Jones KB, Jordan R (2015) White lesions in the oral cavity: clinical presentation, diagnosis, and treatment. Semin Cutan Med Surg 34(4): 161-170.

7. Greenbreg M, Glick M, Ship J (2008) Burkets Oral Medicine. 12th ed. BC Deker; London, pp. 103-104.

8. Indhumathi S, Rajappa M, Chandrashekar L, Ananthanarayanan $\mathrm{PH}$, Thappa DM, et al. (2016) The HLA-C*06 allele as a possible genetic predisposing factor to psoriasis in South Indian Tamils. Arch Dermatol Res 308(3): 193-199.

9. Gorgen M, Melikoglu M, Ozkan M, Erdem T (2010) Predisposition of allergy in patients with benign migratory glossitis. Oral Surg Oral Med Oral Pathol Oral Radiol Endod 110(4): 470-474.

10. Zargari $O$ (2006) the prevalence and significance of fissured tongue and geographical tongue in psoriatic patients. Clin Exp Dermatol (31)2: 192195.

11. Neville B, Damm D, Allen C, Bouquot J (2002) Oral\& Maxillofacial Pathology. 7th ed. W.B Sanders co; London, pp. 677-679.

12. Redman RS, Vance FL, Gorlin RJ, Peagler FD, Meskin LH (1966) Psychological component in the etiology of geographic tongue. J Dent Res 45: 1403-1408.

13. Gonzaga HF, Chaves MD, Gonzaga LH, Picciani BL, Jorge MA, et al. (2015) Environmental factors in benign migratory glossitis and psoriasis: retrospective study of the association of emotional stress and alcohol and tobacco consumption with benign migratory glossitis and cutaneous psoriasis. J Eur Acad Dermatol Venereol 29(3): 533-536.

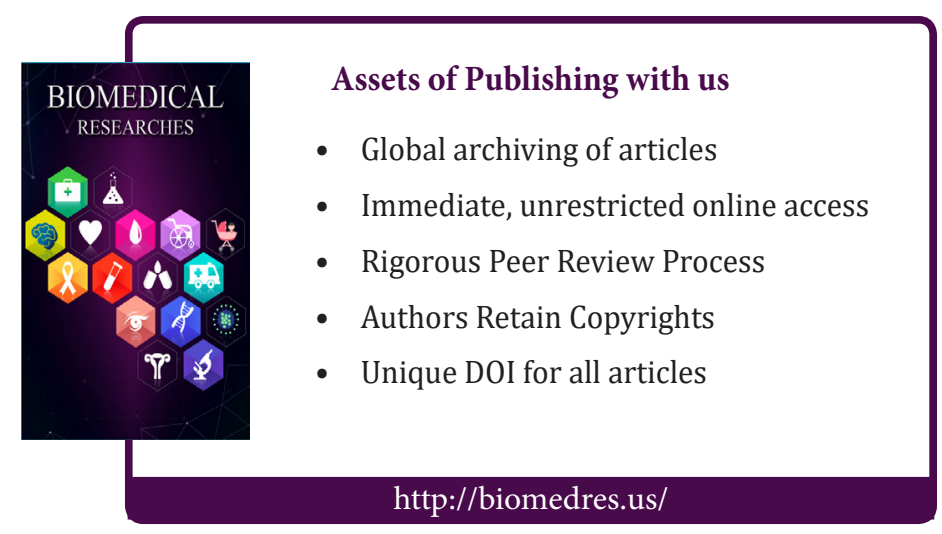

